\title{
VARIATIONAL WAVE FUNCTIONS OF A VORTEX IN CYCLOTRON MOTION
}

\author{
JIAN-MING TANG \\ Department of Physics, University of Washington, \\ P. O. Box 351560, Seattle, WA 98195-1560, USA \\ E-mail: jmtang@u.washington.edu
}

\begin{abstract}
In two dimensions the microscopic theory, which provides a basis for the naive analogy between a quantized vortex in a superfluid and an electron in an uniform magnetic field, is presented. A one-to-one correspondence between the rotational states of a vortex in a cylinder and the cyclotron states of an electron in the central gauge is found. Like the Landau levels of an electron, the energy levels of a vortex are highly degenerate. However, the gap between two adjacent energy levels does not only depend on the quantized circulation, but also increases with the energy, and scales with the size of the vortex.
\end{abstract}

It has become common wisdom to treat a quantized vortex in a two-dimensional superfluid like an electron in an uniform magnetic field. This analogy is based on the fact that the dynamics of a vortex in an ideal classical fluid is determined by the Magnus force and the effective mass of the vortex. 3 Like the Lorentz force, the Magnus force is a transverse force whose magnitude is proportional to the velocity of the vortex relative to the background fluid. The effective mass is related to the fluid displaced by the motion of the vortex, and is sensitive to the structure of the vortex core. The trajectory of a classical vortex therefore consists of a circular orbit around a guiding center. However, there is no quantum theory starting from first principles which supports the existence of such cyclotron motion for a quantized vortex in a superfluid. Experimental evidence for these collective modes has been shown in a more complicated situation, where the cyclotron motion is coupled to the standing wave along the vortex filament 3 .

It is the purpose of the present study to provide a microscopic theory for this phenomenological analogy, and show that the rotational states of a quantized vortex form highly degenerate energy levels similar to the Landau levels in the integer quantum Hall effect. In order to describe these rotational states, which are collective modes in a many-body system, I construct variational wave functions. It was suggested by Hill and Wheeler 5 and also by Redlich and Wigner 5 that the wave function of a collective excitation in a nucleus could be constructed by integrating out the surface variables, which specify the position and the orientation of the nucleus. A quasi-harmonic oscillator wave function was proposed for the weighting function of the integration. Peierls, Yoccoz and Thouless later pointed out that the weighting function could be determined from the variational principle. plying their scheme on a vortex, I calculate the energy spectrum and the weighting functions, which can be thought as the single-particle "wave functions" of a vortex, in a finite system of dilute bosons. The results are found to be consistent with the analogy, except that the energy gap between two neighboring levels is not a constant, and scales logarithmically with the size of the vortex.

I start with a system of $N$ bosons in a disk geometry with radius $R$. The Hamil-

vortex-MBX-0.2: submitted to World Scientific on November 3, 1999 
tonian contains interactions and a confining potential representing the boundary of the system. A static vortex, with a quantized circulation $h / m$, located at a certain point $\mathbf{r}_{0}$ in space can be described by a Feynman wave function 9

$$
\Psi_{\mathbf{r}_{0}}=\left[\prod_{j=1}^{N} g\left(\left|\mathbf{r}_{j}-\mathbf{r}_{0}\right|\right) e^{i \phi\left(\mathbf{r}_{j}, \mathbf{r}_{0}\right)}\right] \Psi_{\mathrm{gs}}\left(\mathbf{r}_{1}, \cdots \mathbf{r}_{N}\right),
$$

where $g(r)$ is a cut-off function to regularize the kinetic energy near the core, and $\phi\left(\mathbf{r}, \mathbf{r}_{0}\right)$ is the velocity potential. An image vortex with an opposite circulation is present at $\left(R / r_{0}\right)^{2} \mathbf{r}_{0}$ so that the normal component of the velocity field vanishes at the boundary. The wave functions representing the rotational states are constructed as linear combinations of Feynman wave functions centered at different positions,

$$
\Psi_{n, l}=\int d^{2} \mathbf{r}_{0} f_{n, l}\left(r_{0}\right) e^{i l \theta_{0}} \Psi_{\mathbf{r}_{0}}\left(\mathbf{r}_{1}, \cdots \mathbf{r}_{N}\right),
$$

where $n$ is the principal quantum number, $l$ is the angular momentum, and $f_{n, l}\left(r_{0}\right)$ is the radial part of the weighting function that needs to be determined. This trial function $\Psi_{n, l}$ possesses the proper symmetry of a quantum state because it does not contain the vortex position $\mathbf{r}_{0}$ explicitly. The single-particle aspect of the collective motion is represented by $f_{n, l}\left(r_{0}\right) e^{i l \theta_{0}}$. Minimizing the energy integral leads to an integral equation for $f_{n, l}\left(r_{0}\right)$,

$$
\int d r_{0}\left[K_{l}\left(r_{0}^{\prime}, r_{0}\right)-\varepsilon_{n, l} J_{l}\left(r_{0}^{\prime}, r_{0}\right)\right] f_{n, l}\left(r_{0}\right)=0,
$$

where $J_{l}$ is the overlap between two Feynman wave functions separated by a finite distance, $K_{l}$ is the same overlap weighted by the kinetic energy, and $\varepsilon_{n, l}$ is the variational energy relative to the ground-state energy. The interaction potential is hidden inside the ground state, and does not show up explicitly in Eq. (3). In the simplest approximation, in which the cut-off function and the ground state are both set to unity, a calculation on the overlap integrals gives

$$
\begin{aligned}
J_{l}\left(r_{0}^{\prime}, r_{0}\right) & =r_{0} \int d \Delta \theta_{0} m S_{l}\left(r_{0}^{\prime}, r_{0}, \Delta \theta_{0}\right), \\
K_{l}\left(r_{0}^{\prime}, r_{0}\right) & =r_{0} \int d \Delta \theta_{0}\left(\ln \frac{2 R}{d}+\beta\right) S_{l}\left(r_{0}^{\prime}, r_{0}, \Delta \theta_{0}\right), \\
S_{l}\left(r_{0}^{\prime}, r_{0}, \Delta \theta_{0}\right) & =\exp \left\{-\left(\frac{1}{2} \ln \frac{2 R}{d}+\alpha\right) d^{2}-i\left[r_{0} r_{0}^{\prime} \sin \Delta \theta_{0}-(N-l) \Delta \theta_{0}\right]\right\},
\end{aligned}
$$

where $\alpha=1.0074, \beta=-0.30685, d=\left|\mathbf{r}_{0}-\mathbf{r}_{0}^{\prime}\right|, \Delta \theta_{0}=\theta_{0}^{\prime}-\theta_{0}$, and $m$ is the mass of the particle. The unit length is set to be the interatomic spacing $\sigma \equiv R / \sqrt{N}$, and $\hbar$ is set to unity. The integral equation, Eq. (3) with Eqs. (4)-(6), is exactly solvable as an eigenvalue problem for $J_{l}^{-1} K_{l}$. It is found numerically that the variational energy $\varepsilon_{n, l}$ is independent of the angular momentum $l$. Numerical solutions of $\varepsilon_{n}$ and $f_{n, l}\left(r_{0}\right)$ are summarized in Fig. 1 and Fig. 2.

The motion of a vortex can now be understood by comparing the weighting functions in Fig. 1 to the eigenfunctions of the Hamiltonian of an electron moving

vortex-MBX-0.2: submitted to World Scientific on November 3, 1999 


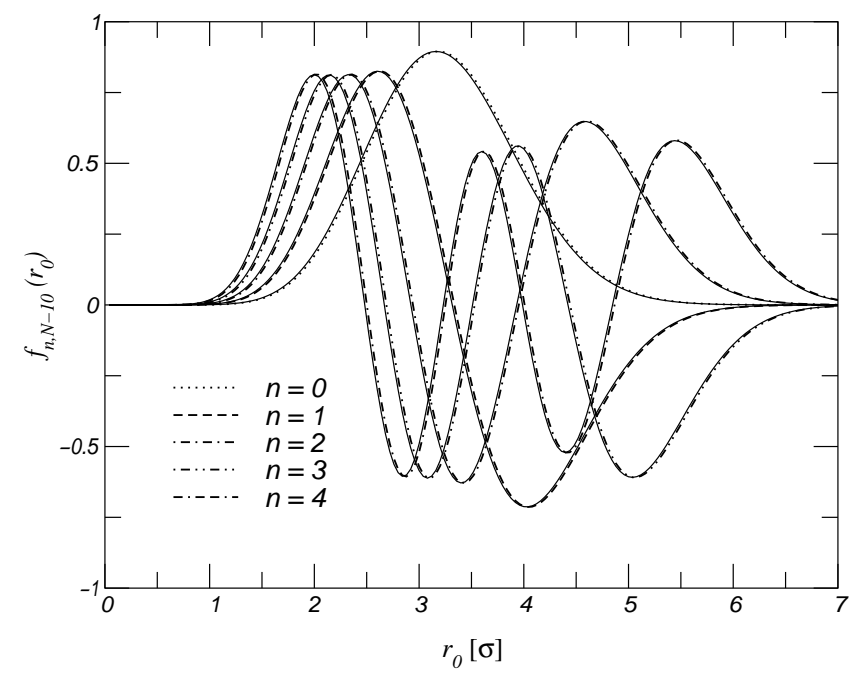

Figure 1. An example of the weighting functions, shown by the solid lines and normalized as $\int d r_{0}\left|f\left(r_{0}\right)\right|^{2}=1$, representing the vortex states with angular momentum $l=N-10$. The dotted lines show the corresponding radial wave functions of an electron in the central gauge with angular momentum $l_{z}=-10$.

in the $x-y$ plane with an uniform magnetic field $B$ pointing in the $z$ direction,

$$
H=\frac{1}{2 m}(\mathbf{p}-e \mathbf{A})^{2}=\frac{\mathbf{p}^{2}}{2 m}+\frac{m}{2}\left(\frac{\omega}{2}\right)^{2}\left(x^{2}+y^{2}\right)+\frac{\omega}{2} L_{z},
$$

where $\omega=|e| B / m$ is the cyclotron frequency, $L_{z}$ is the angular momentum operator perpendicular to the plane, and the central gauge $\mathbf{A}=(-y B / 2, x B / 2)$ is chosen so that $L_{z}$ commutes with the Hamiltonian. The radial wave function $\psi_{n, l_{z}}(r)$ in the $n$-th Landau level with angular momentum $l_{z}$ is identical to the weighting function $f_{n, N+l_{z}}\left(r_{0}\right)$ up to a proper normalization, if the unit length is chosen as $\sqrt{2 \hbar / m \omega}$. This one-to-one correspondence immediately tells us that the motion of a vortex is indeed cyclotron-like, and the degeneracy of each energy level in Fig. 2 is one per $\pi$ unit area, since the degeneracy of a Landau level is $e B / h$ per unit area.

The energy spectrum of a vortex, however, is different from the spectrum of an electron. The Hamiltonian in Eq. (7) basically describes a particle in a harmonic potential in the rotating frame, and has a fixed energy scale $\hbar \omega$ set by the magnetic field. In Fig. 2 the level spacing of a vortex is increasing with energy, and scales down logarithmically with the size of the vortex. Both these features are attributed to the long-ranged phase coherence, which causes the logarithmic size-dependence of the overlap in Eq. (6) between two Feynman wave functions. The small difference among level spacings represents a breakdown of the analogy because one cannot simply add an attractive potential in Eq. (7) to mimic the spectrum due to the fact that the degeneracy of the Landau level will be broken by this extra interaction. The scaling of the level spacing can still be incorporated into the single-particle picture by requiring that the effective mass of the vortex scales with the size accordingly.

vortex-MBX-0.2: submitted to World Scientific on November 3, 1999 




Figure 2. The energy spectrum corresponding to the cyclotron motion of a vortex relative to the lowest energy level. The dotted lines show the scaling of each energy level with the system radius $R$ ranging from 20 to $10^{6}$. The lowest energy level approximately scales as $\ln (R / \sigma)$ corresponding to the formation energy of a static vortex.

In conclusion, I have presented a quantum theory to support the picture that a quantized vortex behaves like an electron in a magnetic field. Vortex states with different angular momenta form highly degenerate levels.

\section{Acknowledgments}

It is a pleasure to thank David Thouless for initiating the idea of this work and stimulating discussions. The author is also grateful to Ping Ao for valuable comments and suggestions. This work was partially supported by NSF DMR-9815932.

\section{References}

1. M. Hatsuda, S. Yahikozawa, P. Ao, and D. J. Thouless, Phys. Rev. B 49, 15870 (1994).

2. S. H. Lamb, Hydrodynamics (The University Press, Cambridge, 1932).

3. R. A. Ashton and W. L. Glaberson, Phys. Rev. Lett. 42, 1062 (1979).

4. K. Karrai et al., Phys. Rev. Lett. 69, 355 (1992).

5. D. L. Hill and J. A. Wheeler, Phys. Rev. 89, 1102 (1953).

6. M. G. Redlich and E. P. Wigner, Phys. Rev. 95, 122 (1954).

7. R. E. Peierls and J. Yoccoz, Proc. Roy. Soc. A 70, 381 (1957).

8. R. E. Peierls and D. J. Thouless, Nucl. Phys. 38, 154 (1962).

9. R. P. Feynman, in Progress in Low Temperature Physics, edited by C. J. Gorter (Elsevier Science Publishers B.V., Amsterdam, 1955), Vol. 1, Chap. 2, pp. 1753. 